\title{
Sino-Maxillary Mucormycosis of Iatrogenic etiology in an immunocompetent patient - Importance of early diagnosis and prompt management
}

\section{Priya Jeyaraj}

${ }^{1}$ Classified Specialist (Oral \& Maxillofacial Surgery), Commanding Officer, Military Dental Centre (Gough Lines), Secunderabad, India

*Corresponding Author: Priya Jeyaraj, Classified Specialist (Oral \& Maxillofacial Surgery), Commanding Officer, Military Dental Centre (Gough Lines), Secunderabad, India.

Received date: March 11, 2021: Accepted date: March 29, 2021: Published date: April 05, 2021

Citation: Jeyaraj P. (2021) Sino-Maxillary Mucormycosis of Iatrogenic etiology in an immunocompetent patient - Importance of early diagnosis and prompt management; J Clinical Otorhinolaryngology 3(2); DOI: 10.31579/2692-9562/029

Copyright: (C) 2021, Priya Jeyaraj. This is an open access article distributed under the Creative Commons Attribution License, which permits unrestricted use, distribution, and reproduction in any medium, provided the original work is properly cited.

\begin{abstract}
:
Sino-maxillary mucormycosis is an opportunistic, aggressive, invasive and fulminant fungal infection, which has the ability to rapidly cross anatomical barriers, causing extensive hard and soft tissue destruction, leading to significant cosmetic deformity, functional debility and morbidity. It usually affects susceptible, debilitated or immunocompromised individuals. A comprehensive Review Paper is presented, with a descriptive report on an unusual Case presentation, involving the dentoalveolar complex which thereafter invaded the maxillary antrum, in an otherwise healthy, adult patient with nil comorbidities. A probable intraoral portal of entry, and a likely iatrogenic etiopathogenesis resulting from inoculation of the fungus or its spores during dental extractions carried out in an unsterile setting, has been considered and elucidated. An emphasis has been placed on according a high index of suspicion in diagnosis of this fungal infection even in immunocompetent and healthy adults, presenting with a seemingly simple, deceptively indolent odontogenic infection. The importance of careful, albeit rapid differential diagnosis, and a prompt and aggressive medical-surgical treatment protocol instituted immediately following a confirmatory diagnosis, helps in limiting the spread and degree of destruction which can be caused by this potentially lethal fungal infection.

Keywords: mucormycosis; zygomycosis; phycomycosis; surgical debridement; amphotericin b; platelet rich fibrin $(\mathrm{PRF})$
\end{abstract}

\section{Introduction:}

Invasive fungal infections, otherwise referred to as mycoses, are uncommon, however when they do occur, they can be quite destructive, functionally debilitating, aesthetically deforming and devastating to patients. Mucormycosis is a rare opportunistic infection [1], which represents the third most common angio-invasive fungal infection, following candidiasis and aspergillosis, and is considered one of the most important medical complications in diabetics and immunocompromised patients. Mucormycosis, also referred to as Phyomycosis or Zygomycosis, is caused by a saprophytic aerobic fungus, belonging to the Class Phycomycetes / Zygomycetes; Order Mucorales; Family Mucoraceae and Genera Rhizopus, Mucor and Absidia [1, 2]. These opportunistic pathogens are ubiquitous organisms, existing in the environment, soil, air, food, bread molds, decaying vegetations, compost piles, animal excreta, and play a pivotal role in the cycle of decomposition in the natural world. Although majority of these pathogenic fungi require oxygen, they are capable of growing in anaerobic and microaerophilic conditions as well [2]. This organism is frequently found to colonize the oral mucosa, nasal mucosa, paranasal sinuses and pharyngeal mucosa of asymptomatic patients [3, 4].
These fungi do not usually cause disease in healthy people with intact immune systems, however, patients with predisposing immunosuppressive conditions may develop this invasive fungal disease [5]. These conditions include diabetes mellitus, renal failure, liver cirrhosis, malignancies, intravenous drug abuse, malnutrition states, stem cell transplants, immunosuppression, systemic corticosteroid therapy, neutropenia and hematological malignancies like multiple myeloma, leukemia and lymphoma [6-8]. It is likely that the hyperglycaemia state in diabetics stimulates fungal growth, and the reduction in chemotaxis and phagocytic efficiency in immunosuppressed conditions permit these otherwise innocuous organisms to proliferate. In the past decade, cases of mucormycosis have even been described in immunocompetent/otherwise healthy individuals, with no identifiable risk factors $[9,10]$.

Mucormycosis generally presents as an acute infection manifesting in rhinocerebral, pulmonary, gastrointestinal, cutaneous, or disseminated forms [11]. These opportunistic infections occur when the causative fungal organisms, to which we are frequently exposed [10], gain entry into the body due to a decrease in host defenses or through an invasive portal. The infection usually begins in the upper nasal turbinate or paranasal sinuses due to inhalation of fungal spores, or less commonly in 
the palate or pharynx. It can spread to orbital and intracranial structures either by direct invasion or through the blood vessels. The fungus invades the arteries leading to thrombosis that subsequently causes necrosis of hard and soft tissues. Tissue necrosis, often a late sign, is a hallmark of mucormycosis, resulting from angio-invasion and vascular thrombosis [11].

Rhinocerebral mucormycosis [12] is capable of producing widespread involvement of the oral cavity, maxilla, palate, nose, paranasal sinuses, orbits and central nervous system. Early symptoms of this disease include facial cellulitis, periorbital edema and nasal inflammation, followed by widespread tissue necrosis. Failure of prompt medical and surgical intervention may lead to cerebral spread, cavernous sinus thrombosis, septicemia and multiple organ failure lending to high morbidity and mortality.

The case of Sino-maxillary oral mucormycosis reported in this paper, was out of the ordinary in its presentation on two accounts. Firstly, its development following a dental extraction, which was indicative of the dental extraction site as the likely but unusual portal of entry of the causative pathogen. Secondly, it's surprising occurrence in an immunocompetent and healthy adult, which added to the diagnostic and therapeutic dilemma of the case.

\section{Case report:}

A 62-year-old male patient (Figure 1) reported with the complaint of nonhealing extraction sockets of upper molar teeth on the right side, which had been extracted 3 months ago. He also complained of an occasional foul smelling discharge from the area and a troublesome nasal regurgitation of orally ingested fluids. In addition, he had also been experiencing a heaviness and dull ache in the region of the right maxillary sinus, which radiated to the right temple.
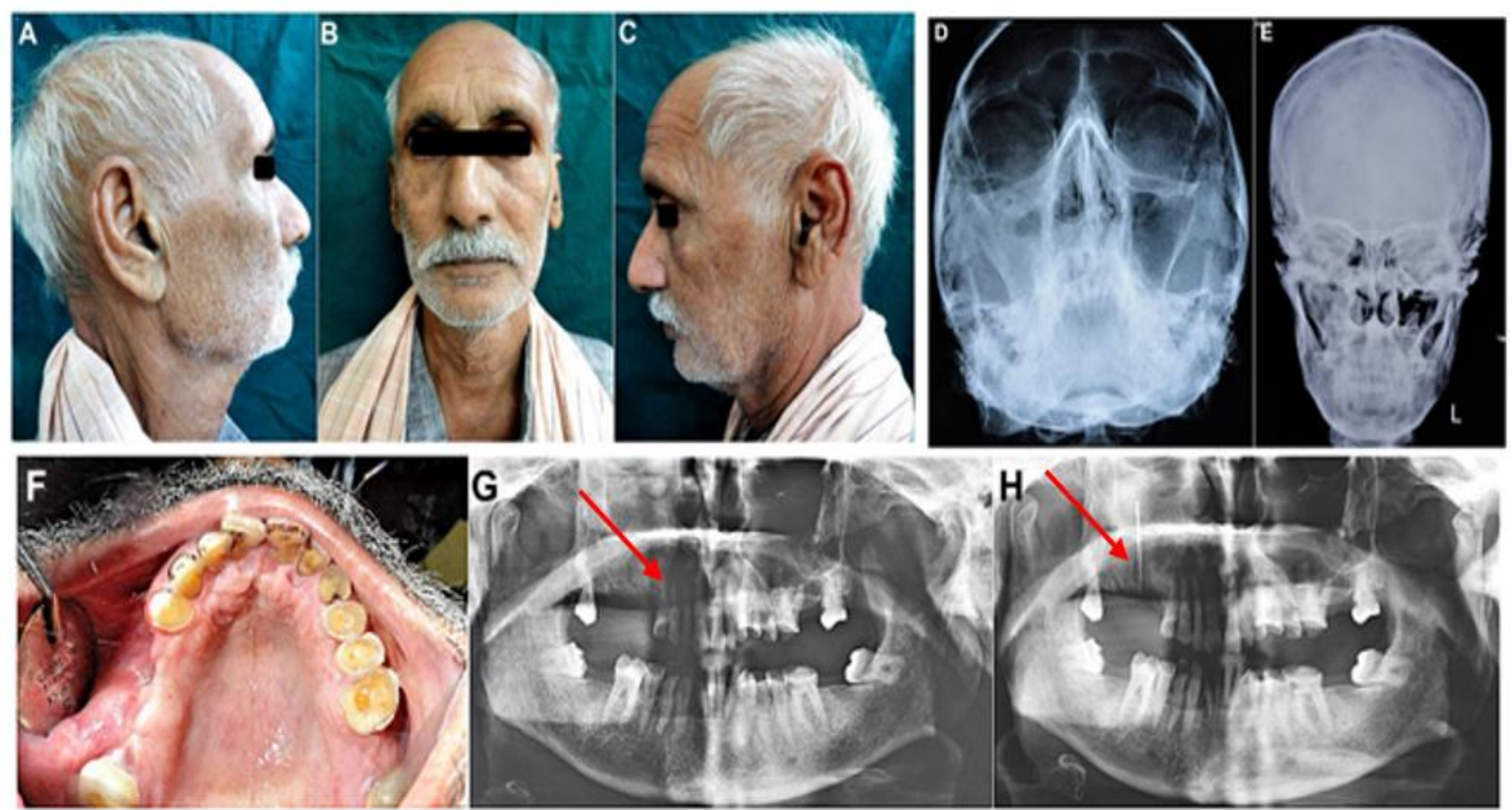

Figure 1: A 62-year-old healthy, immunocompetent patient who presented with a persistent post-extraction pain in the upper right quadrant, vague and dull pain and a feeling of heaviness in the region of the right maxillary sinus, accompanied by nasal regurgitation of orally ingested fluids via an oroantral communication, for the past three months. The complaints had developed following multiple upper dental extractions that had been carried out in an non-sterile setting at a rural clinic. (D, E) Radiographs (Para Nasal Sinuses view and Postero-Anterior view of skull) revealed a distinct haziness and opacification of the right maxillary antrum. (F) Intraoral examination revealed poor oral hygiene and a persisting oroantral fistula in the region of extraction socket of tooth 16. (G) Orthopantomogram revealed irregular bony destruction in the region of right maxillary quadrant and reduced periodontal support of the anterior teeth in the region. $(\mathrm{H}) \mathrm{A}$ Gutta percha point placed in the incompletely healed extraction socket of 16 , could be seen entering well within the right maxillary antrum, confirming the presence of an oroantral communication.

History revealed that he had been to a dental surgeon in a rural area three months ago for evaluation of several loose upper teeth. The mobility of multiple upper teeth was assumed to be due to their poor periodontal condition and lack of adequate tooth support resulting from chronic periodontitis. Hence, multiple dental extractions had been carried out in one sitting, without any flap reflection or osseous surgery. The patient's post extraction course was protracted and painful which made him seek management by us.

On examination, the patient was afebrile with no visible swelling or features of acute inflammation (Figure 1 A-C). He was partially edentulous with multiple missing maxillary $(17,16,15,27)$ as well as mandibular (37 and 47) teeth. He had poor oral hygiene, with generalized periodontitis involving the remaining standing teeth. There was an oroantral fistula in the edentulous right molar region (Figure 1F), presence of which was confirmed by inserting a Gutta percha point into the defect and taking radiographs thereafter (Figure $1 \mathrm{G}, \mathrm{H}$ ). Purulent material was seen draining intraorally though the fistula. Nasal regurgitation of water was also observed when he was asked to rinse his mouth, further confirming the oro-antral communication. The remaining teeth $(18,11,12,13$ and 14) of the right upper quadrant were tender on percussion and exhibited Grade 3 mobility. The palatal mucosa appeared normal. Clinical presentation was suggestive of right maxillary sinusitis in relation to a residual oroantral fistula, which had developed following multiple dental extractions. 
An Orthopantomogram (Figure 1G, H) showed irregular bony destruction presenting as a moth-eaten appearance, of the right maxillary alveolar region in relation to teeth $11,12,13$ and 14 , with clearly evident loss of their bony support. There was also noted a haziness and radiopacity of the right maxillary antrum, while the left side appeared normal. Paranasal Sinuses (PNS / Water's) view and Postero-anterior (PA) view radiographs of the skull (Figure $1 \mathrm{D}, \mathrm{E})$ too demonstrated a diffuse haziness and opacification of the entire right maxillary antrum. Differential diagnosis included acute Maxillary sinusitis, post-extraction bacterial / fungal maxillary infection and Osteomyelitis of the right maxilla.

The patient did not give any history of a possible immunosuppressed state such as Diabetes mellitus, organ transplantation, immunosuppressive therapy, malignant disease, chemotherapy, irradiation, etc. A complete work up was carried out to assess the immunological status of the patient and to check for the presence of any comorbidities, such as leukemia, malignancy, chronic obstructive pulmonary disease (COPD), etc., all of which were ruled out. A full workup of serum glucose, electrolytes, blood chemistries and blood gases was carried out and Diabetes mellitus was ruled out. Leukocytosis was noted with larger numbers of lymphocytes, suggestive of a chronic infection.

A scraping was taken from within the oro-antral fistula, including some tissue curetted from within the right maxillary sinus, for carrying out histopathological examination (Figure 2). Light microscopic evaluation of Haematoxylin and Eosin stained sections of the biopsy specimen revealed ulcerated pseudostratified sinus epithelium with underlying edematous chronically inflamed stroma (Figure $2 \mathrm{~A}, \mathrm{~B}$ ) with numerous large colonies of broad, ribbon-like aseptate fungal hyphae branching at acute and right angles; and their spores (Figure $2 \mathrm{C}-\mathrm{E}$ ). Vascular inhabitations by the hyphae were also noted within the large areas of necrosis. To further confirm the fungal infection, Fungal silver stain, namely the Grocott and Gomori methenamine silver (GMS) stain [14, 19] was employed for the remaining tissue specimen. The fungal cell wall appeared dark brown, almost black, while the surrounding tissue appeared green (Figure $2 \mathrm{~F}$ ). This confirmed the diagnosis of Mucormycosis (Phycomycosis, Zygomycosis).

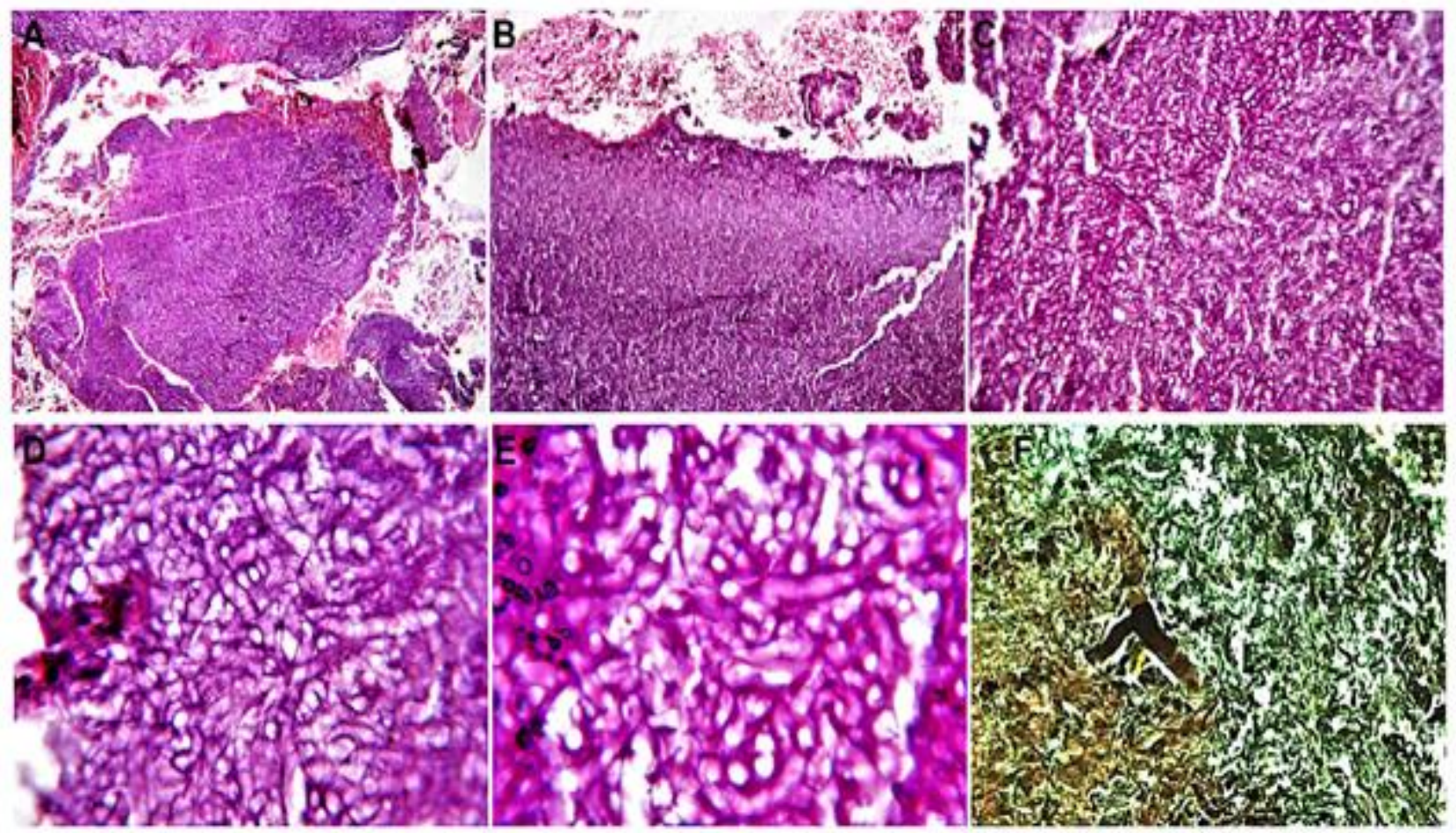

Figure 2: Figure 1. Histological analysis of tissue scrapings taken from the oroantral fistula and specimen fragments of the antral lining, confirming the diagnosis of Mucormycosis (Magnifications X50, X80, X100, X150). (A, B) (Haematoxylin \& Eosin X 50) showing ulcerated ciliated, pseudostratified sinus epithelium with underlying edematous and inflamed stroma, chronic inflammatory cell infiltrate, thrombosed vascular channels, focal areas of haemorrhage and large areas of necrotic tissue with evident cellular degeneration and debris. (C, D, E) (Haematoxylin \& Eosin X 80, X100, X150) Multitudes of large colonies of aseptate fungal hyphae branching at right angles $\left(90^{\circ}\right)$, and their spores. The fungal colonies comprised of non-pigmented, wide (10 to $20 \mu \mathrm{m}$ ), thin-walled, ribbon-like hyphae with few septations (pauciseptate) and right-angle branching. A few vascular channels were seen with inhabitation by the fungal hyphae and spores. (F) Grocott methenaminesilver staining (magnification X150) of biopsy tissue specimen showing dark brown fungal hyphae with a right-angle branching, in a greenish tissue background, confirming the fungal organism to be of the order Mucorales.

A CT Scan was performed for evaluation of the extent of involvement (Figure 3). 3-D reformatted images showed irregular destruction of the maxillary bone in the right upper quadrant, which included the alveolar region as well as the anterolateral wall of the right maxillary antrum (Figure 3A). On coronal and sagittal sections (Figure 3B, C), the antral lining on the right side showed radio-dense, polypoid thickenings, giving it a corrugated appearance; and the entire maxillary antrum appeared homogeneously opacified, while the contralateral maxillary antrum appeared uninvolved. The medial wall of the right antrum /lateral wall of the nasal cavity appeared to pushed medially, however there was no breach or bony destruction. The nasal cavities, ethmoid and frontal sinuses, orbital floor and the left maxillary antrum appeared normal and uninvolved (Figure D, E). 

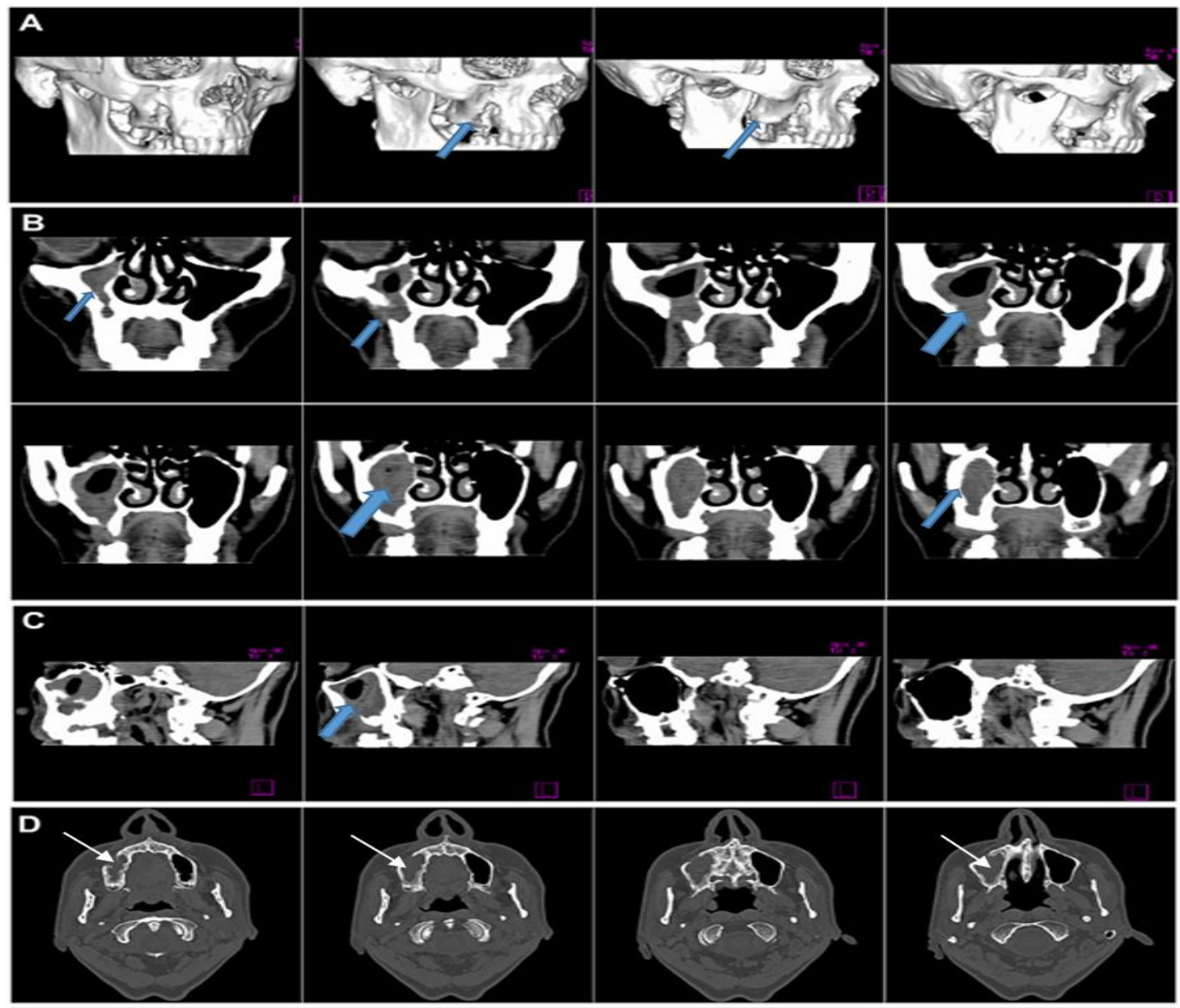
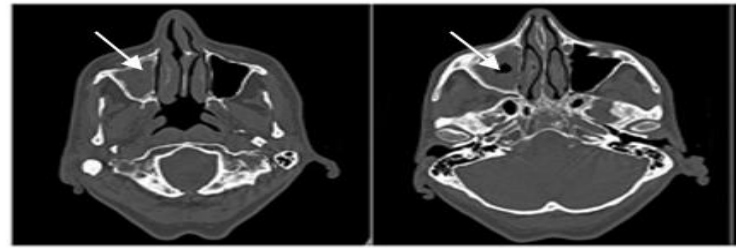

Figure 3: Non-contrast computed tomographic (NCCT) scans of the maxillofacial region. (A) 3-D reformatted images showing irregular bony destruction in the right posterior maxillary dentoalveolar region, extending to involve the lateral wall of the maxillary antrum, creating a wide oroantral communication via the necrosed and enlarged extraction socket of 16. (B) Coronal sections showing a distinct thickening and polypoid enlargements in the schneiderian membrane of the right maxillary sinus, giving it a corrugated, radio-dense appearance. The breach in the lateral sinus wall leading to the oroantral fistula is clearly evident. More posteriorly, the entire antrum appears to be diffusely and homogeneously opacified and obliterated by soft tissue mass. The ethmoid sinuses appear uninvolved. No bony disruption or breach detected in the orbital floor or lateral nasal wall, with nil evidence of orbital or nasal cavity extension. (C) Sagittal sections through the right and left maxillary antra showing a contrasting appearance of their interior, which appears diffusely opacified and obliterated on the right, whereas clear and uninvolved on the left side. (D) Axial sections showing obliteration of the right maxillary antrum by a homogenously enhancing soft tissue density, destruction of portions of its anterolateral wall, with uninvolved antral floor, palatal bone, and antral roof / orbital floor. The lateral wall of the right nasal cavity appears to be pushed medially,

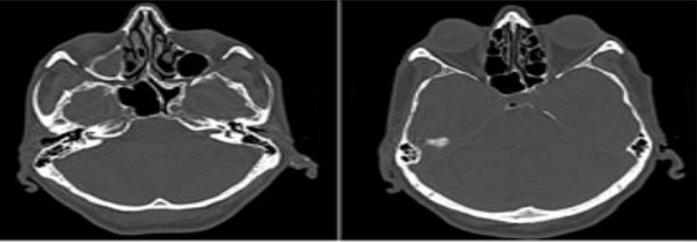

however no breach in the bone is detectable. The contralateral / left maxilla, orbits and ethmoid sinuses appear uninvolved.

Treatment planned for the patient aimed at an aggressive medico-surgical combination treatment protocol [13], comprising of antifungal chemotherapy coupled with thorough surgical local debridement of all necrotic tissue, to completely eradicate the fungal pathogen. Surgical intervention was planned under general anaesthesia, and included extraction of all remaining teeth of the right maxillary quadrant; removal of the necrotic soft tissue slough and sequestrectomy of loose, necrosed bone in the region; removal of the antral polypoid enlargements; surgical debridement of the entire right maxillary antrum with complete extirpation of the sinus lining, antral curettage and lavage; antral irrigation with an antifungal agent; placement of fresh autologous platelet rich fibrin within the surgical defect; and closure of the oroantral communication.

The patient was operated under general anaesthesia (Figure 4). A full thickness buccal mucoperiosteal flap was raised, exposing the entire right dentoalveolar complex and anterolateral wall of the maxillary antrum (Figure 4 A-C). The mucosal lining of the oroantral fistula was carefully excised. All loose bone fragments were removed from the region and necrosed margins of bone were nibbled using bone rongeurs. Extraction of the remaining standing teeth of the upper right quadrant was carried 
out (Figure 4 D, E). All necrotic material (Figure 4 F-H) was curetted out and sequestered bone pieces were carefully removed, which led to exposure of the interior of the right maxillary antrum. The antral lining was found to be thick and fibrous, with multiple polypoid growths, all of which were carefully curetted out (Figure 4 I-L). Maxillary sequestrectomy and debridement, extirpation of the entire antral lining, antral curettage and lavage were carried out (Figure M-O). A lipid preparation of Amphotericin B was used to irrigate the maxillary antrum to destroy any remaining fungal spores and to discourage any subsequent fungal proliferation. Fresh autologous platelet rich fibrin was prepared intraoperatively using a centrifuge, and was placed within the surgical site to encourage subsequent soft and hard tissue healing and regeneration (Figure 4P-R). The mucoperiosteal flap was then replaced and the wound closed. (Figure $4 \mathrm{~S}, \mathrm{~T}$ ).
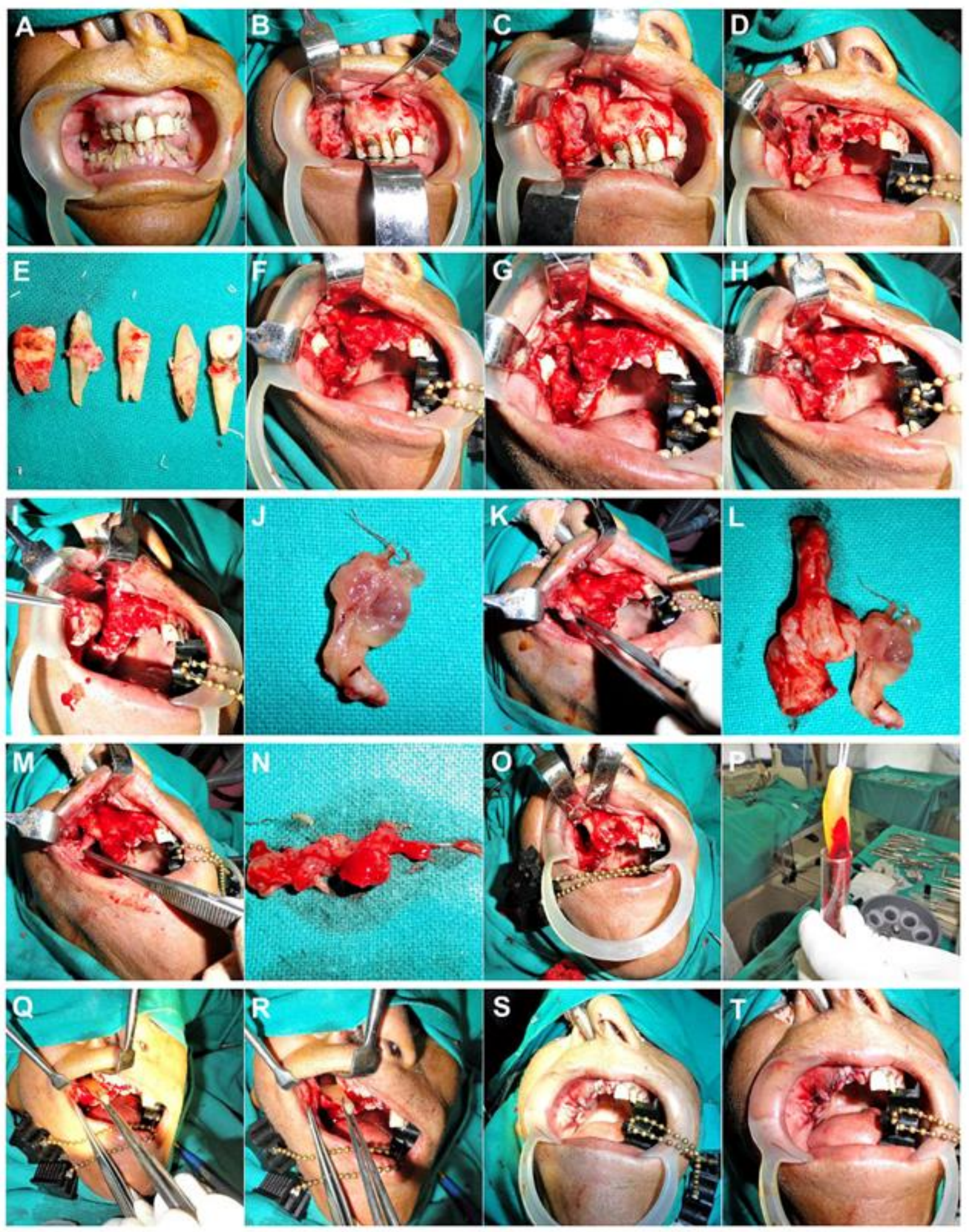

Auctores Publishing - Volume 3(2)-029 www.auctoresonline.org 
Figure 4: Intraoperative photographs. (A-C) Long crevicular incision extending from tooth 21 to 18 placed, with vertical buccal relieving incisions at either end. Full thickness mucoperiosteal flap raised exposing the dentoalveolar complex and anterolateral wall of the right maxilla. Irregular bone destruction and the wide oroantral communication seen in the 16, 17 region. (D, E) Mucous membrane lining oroantral fistula, excised followed by extraction of remaining teeth of the right upper quadrant. (F-H) Greyish white eschar and necrotic material seen in the posterior part of operative field, which was carefully curetted out. (I-L) Antral polyp-like soft tissue masses excised from within the right maxillary antrum. (M, N) Sinus lining extirpated, followed by thorough antral curettage and lavage to ensure elimination of all tissue and necrotic debris likely to contain fungal colonies and spores. (O) Necrotic margins of bone nibbled from the walls of the antrum, thus completing the sequestrectomy and debridement. A final irrigation of the antrum was carried out with a lipid solution of Amphotericin-B. (P-R) $20 \mathrm{ml}$ of fresh autologous Platelet Rich Fibrin (PRF) prepared intraoperatively, using a centrifuge. PRF carefully placed within the surgical site, to encourage wound healing, and hasten subsequent bone and soft tissue regeneration. (S, T) Flap replaced and watertight wound closure carried out using interrupted Vicryl 3-0 sutures.

Postoperative recovery was smooth and uneventful. The patient was administered a single daily dose of liposomal Amphotericin B $(1 \mathrm{mg} / \mathrm{kg}$ body weight/day) as an intravenous infusion in $100 \mathrm{ml}$ of $5 \%$ dextrose, over 1 to 2 hours, for a period of two weeks. Renal functions (blood urea and creatinine levels) of the patient were monitored carefully during this period, due to the known nephrotoxicity of the drug. By the end of the week, signs of healthy granulation tissue and satisfactory wound healing was observed (Figure 5).

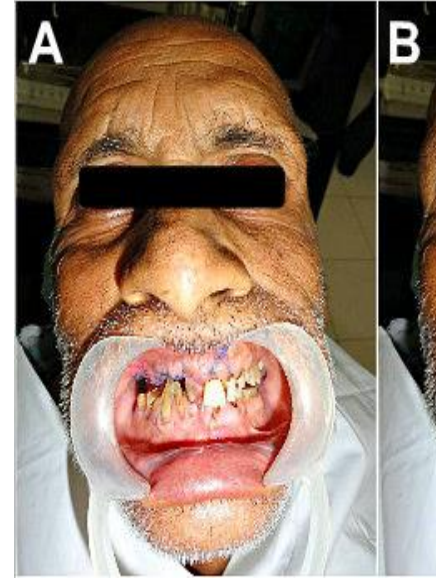

Figure 5: Smooth and uneventful healing of the operated site. Complete closure of the oroantral fistula and resolution of the Sino-maxillary mucormycosis achieved by the combination protocol of aggressive surgical debridement and antifungal chemotherapy.

The patient was discharged from the hospital on the fifteenth postoperative day, after placing him on oral Fluconazole (50 mg twice a day) for one week [14]. All intraoral sutures were removed on the tenth postoperative day. Successful closure of the oroantral fistula was achieved and there was observed no wound dehiscence. The patient was followed up for 13 months, both clinically as well as by taking serial radiographs. There was observed good regeneration of the anterolateral wall of the right maxilla and no recurrence of the fungal infection.

\section{Discussion:}

Mucormycosis is the third invasive mycosis [2, 3] in order of importance after candidiasis and aspergillosis and is caused by fungi of the class Zygomycetes. Based on clinical presentation and the involvement of a particular anatomic site [12], mucormycosis can be divided into at least six clinical categories: (i) rhinocerebral, (ii) pulmonary, (iii) cutaneous, (iv) gastrointestinal, (v) disseminated, and (vi) miscellaneous. Chakrabarti et al. observed that rhino-orbito-cerebral type (44.2\%) was the commonest presentation followed by cutaneous (15.5\%) and renal $(14.0 \%)$ involvement in their retrospective analysis for ten years in India [12]. The overall survival rate of patients with mucormycosis ranges from $50 \%$ to $85 \%$ with a higher survival rate of rhino-cerebral mucormycosis than pulmonary or disseminated forms as the rhinocerebral disease can frequently be diagnosed earlier, and the most common underlying cause, diabetic ketoacidosis, can be readily treated [13]. Notably, if the disease has not penetrated beyond the sinus, the prognosis is much better; indeed, in local sinonasal disease, the mortality has been reported to be less than $10 \%[14]$.

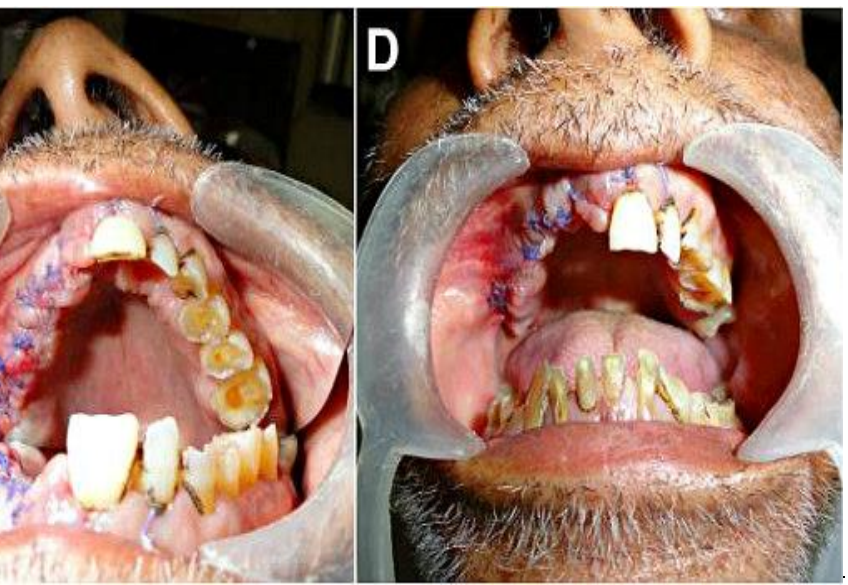

Early detection at the primary site, confirmatory diagnosis based on histopathology and culture, followed by a prompt and aggressive surgical

and antifungal treatment are imperative for successful management of this fulminant fungal infection.

The rhinomaxillary form of the disease, is a subdivision of the rhinocerebral form, and is usually caused due to inhalation of the fungus by a susceptible individual [15]. Inhaled spores cause disease in the upper and lower respiratory tracts of immunosuppressed persons. In immunocompetent or immunosuppressed hosts, spores can be inoculated into the skin and subcutaneous tissues by trauma, needle exposure, or insect bites, and they can be ingested, causing gastrointestinal disease 15, 16]. In rare instances, a dental extraction site may serve as an intraoral portal of entry by the fungus. The case of maxillary mucormycosis described in this report, affecting a healthy and immunocompetent individual, was most likely caused by inoculation of the virulent fungus or its spores at the time of dental extraction, which had probably been carried out in an unhygienic and unsterile setting. Another reason for high propensity of association of mucormycosis with extraction of maxillary posterior teeth can possibly be due to their proximity to the maxillary sinus [11], which often get involved when fungal spores are inhaled through nasal route.

Progression of the maxillary infection to the adjacent anatomical structures such as the nasal cavity, orbit, ethmoid sinuses, cranial cavity etc. can occur easily and rapidly, given the invasive and aggressive nature of this pathogen $[14,15,16]$. Fungal infection of the maxillary sinus can lead on to invasion of the nasal cavity through its lateral wall. Invasion through the maxillary antral roof / orbital floor can lead to orbital involvement, periorbital cellulitis, followed by the onset of conjunctival suffusion, blurry vision and even loss of eyesight. Orbital roof involvement can even serve as a portal of entry to the brain, with the fungus eroding through the floor of the anterior cranial fossa. Spread 
along the fascial planes and through the fascial spaces can produce facial numbness, soft tissue swelling, facial cellulitis, followed by eschar formation and ensuing necrosis of nasofacial region, unless prompt and effective management is undertaken [14]. Advancing infection can also spread from the ethmoid sinus to the orbit, resulting in loss of extraocular muscle function and proptosis with marked chemosis and can quickly result in cavernous sinus thrombosis, carotid artery, or jugular vein thrombosis (Lemierre syndrome) and death [16]. Hence, it is imperative to diagnose this condition quickly and effect prompt and definitive management to avert the possibly disastrous sequelae and complications of this extremely aggressive and invasive infection [17].

In routine maxillofacial practice, intra-oral exposed bone (maxillary necrosis) is generally diagnosed as osteomyelitis. Maxillary necrosis can occur due to bacterial osteomyelitis, herpes zoster, trauma, iatrogenic infections, or fungal infections, such as mucormycosis, aspergillosis, etc. A clinical suspicion of Mucormycosis requires confirmation by radiological and histological examination of a biopsy specimen [18]. Radiographs and CT scans of the maxillofacial region, showing irregular bony destruction accompanied by hyperdensity in the affected paranasal sinuses, schneiderian membrane thickening with or without polypoid growths, are highly suggestive fungal disease [19]. All these findings were observed in the case presented here (Figure 1;3) and included unilateral maxillary sinus inflammatory changes and polypoid mucosal thickening of the antral lining. Radiologically, radio-opaque concretions may be identified in aspergillosis, whereas mucormycosis shows generalized opacification and a diffuse haziness of the sinus as was the case presented [16, 17].

Histopathological confirmation of the biopsy was confirmatory of the diagnosis of mucormycosis in the case presented. Tissue samples from the oroantral fistula and fragments of the lining mucosa of the affected maxillary antrum were screened, by subjecting them first to Haematoxylin and Eosin staining, followed by Grocott and Gomori methenamine silver staining. This dual screening of the tissue sample allowed the fungus as well as the host reaction to be clearly observed, enabling an accurate and rapid confirmatory diagnosis. This facilitated the infection to be managed immediately and effectively, before it involved the adjacent anatomical structures. Characteristic wide, ribbon like, aseptate hyphal elements that branched at right angles, were demonstrable in the scrapings taken from the oroantral fistula as well as in the sections of mucosal lining curetted from the right maxillary antrum. The fungal organisms were observed to be surrounded by extensive necrotic debris. Following surgical management, histological examination of the excised specimen of the extirpated schneiderian membrane as well as the necrotic tissue from the right maxilla, further confirmed the diagnosis.

Four factors or strategies are currently considered critical for eradicating Mucormycosis: rapidity of diagnosis; reversal, removal or reduction of the predisposing/risk factors (drugs or underlying diseases), appropriate surgical debridement of infected tissue, and rapid and aggressive antifungal therapy $[14,20]$. Presence of necrotic bone acts as a nidus of infection, serving as a protective barrier for the organisms, thus preventing the effective action of systemically, administered antifungal drugs [14]. Thrombosis of blood vessels in the region may also reduce antibiotic effectiveness. So, surgical resection of involved hard and soft tissues and thorough debridement of all necrotic material has a very important role to play in effective management of this condition. Empirical parenteral antibiotic therapy with a broad spectrum [17, 18], covering Gram-negative and anaerobic bacteria, such as Cephalosporin and Metronidazole combinations are advocated in addition to antifungal medication, the drug of choice being Amphotericin-B, preferably the lipid formulation because adequate dosage can be delivered with reduced renal toxicity. Debridement of necrotic tissue in combination with medical therapy with antifungal drugs forms the basis of treatment. In consonance with the above management protocol, an aggressive medico-surgical approach was employed in our case as well, with excellent results.

Clinicians have reported the use of up to $7.5-10 \mathrm{mg} / \mathrm{kg} / \mathrm{d}$ to treat mucormycosis, especially in CNS disease [18, 19]; however, the benefit of higher doses is unknown and such doses have a higher risk for nephrotoxicity. Amphotericin B deoxycholate can also be used for the treatment of mucormycosis, especially in settings of cost restraints. In our case, we employed a lipid preparation of amphotericin B administered at a dose of at $1 \mathrm{mg} / \mathrm{kg} / \mathrm{d}$, with good results.

Oroantral fistulas in such cases have usually been reported to have been closed by employing a double layered closure using a pedicled buccal fat pad and buccal advancement flap. buccal pad of fat advancement for closure of the oroantral fistula [21]. In our case, we employed a novel approach by placing fresh autologous PRF in the region of the bone defect at 16 and 17 region, before replacing and suturing the raised buccal mucoperiosteal flap. PRF helped to encourage bone and soft tissue regeneration in the dentoalveolar region, successfully sealing off the oroantral communication.

\section{Conclusion:}

Prognosis of Mucormycosis of the Maxilla and Paranasal sinuses involves a high morbidity and mortality, owing to the rapid spread and extensive necrosis and destruction of contiguous vital structures by this invasive opportunistic pathogen. The severe functional debility and extensive cosmetic deformity it is capable of, makes it a formidable entity to manage effectively. The prognosis of a case definitely improves with a rapid and accurate diagnosis, prompt and early management by an aggressive and coordinated medical cum surgical protocol. High dose antifungal medication in combination with thorough surgical debridement, along with a concomitant reversal of the patient's underlying risk factors and compromising medical conditions and / or immunosuppressive state, if any, comprise the mainstay of management.

\section{References:}

1. S. Viterbo, M. Fasolis, P. Garzino-Demo et al. (2011) Management and outcomes of three cases of rhinocerebral mucormycosis. Oral Surgery, OralMedicine, Oral Pathology, Oral Radiology and Endodontology; 6: 69-74.

2. Kontoyiannis DP, Lewis RE. (2006) Invasive zygomycosis: update on pathogenesis, clinical manifestations, and management. Infect Dis Clin North Am; 20:581-607.

3. Bhansali A, Bhadada S, Sharma A, Suresh V, Gupta A, et al. (2004) Presentation and outcome of rhino-orbital-cerebral mucormycosis in patients with diabetes. Postgrad Med J; 80: 670 $-674$.

4. McNulty JS. (1982) Rhinocerebral mucormycosis: Predisposing factors. Laryngoscope; 92:1140-1143.

5. Lee DG, Choi JH, Choi SM, Yoo JH, Kim YJ, et al. (2002) Two cases of disseminated mucormycosis in patients following allogeneic bone marrow transplantation. J Korean Med Sci; 17:403-406.

6. Jones AC, Bentsen TY, Freedman PD. (1993) Mucormycosis of the oral cavity. Oral Surg Oral Med Oral Pathol; 75:455-460.

7. Kofteridis DP, Karabekios S, Panagiotides JG, Bizakis J, Kyrmizakis D, (2003) Successful treatment of rhinocerebral mucormyc osis with liposomal amphotericin B and surgery in two diabetic patients with renal dysfunction. J Chemother; 15:282286.

8. Serris A, Danion F, Lanternier F. (2019) Disease entities in mucormycosis. J Fungi; 5: 23-25.

9. Michele D. Mignogna, Giulio Fortuna, Stefania Leuci. (2011) Mucormycosis in immunocompetent patients: a case-series of 
patients with maxillary sinus involvement and a critical review of the literature. International Journal of Infectious Diseases; 15: 533-540.

10. Angali RK, Jeshtadi A, Namala VA, Gannepalli A. (2014) Fatal rhino-orbito-cerebral mucormycosis in a healthy individual. $J$ Oral Maxillofac Pathol; 18:460-463.

11. Auluck A. (2007) Maxillary necrosis by mucormycosis: a case report and literature review. Med Oral Pathol Oral Cir Bucal; 12: 360-364.

12. Chakrabarti A, Das A, Sharma A, et al. (2001) Ten years' experience in zygomycosis at a tertiary care centre in India. Journal of Infection; 42: 261-266.

13. Ferguson BJ. (2000) Mucormycosis of the nose and paranasal sinuses. Otolaryngol Clin North Am; 33:349-365.

14. Spellberg B, Edwards Jr J, Ibrahim A. (2005) Novel perspectives on mucormycosis: pathophysiology, presentation, and management. Clin Microbiol Rev; 18:556-569.

15. Mane RS, Watve JK, Mohite AA, Patil BC. (2007) Rhinocerebral mucormycosis: A deadly disease on the rise. Indian J Otolaryngol Head Neck Surg; 59:112-115.

16. Fogarty C, Regennitter F, Viozzi CF. (2006) Invasive fungal infection of the Maxilla following dental extractions in a patient with chronic obstructive pulmonary disease. Journal of the Canadian Dental Association; 72: 149-152.

17. Dhiwakar M, Thakar A, Bahadur S. (2011) Improving outcomes in rhinocerebral mucormycosis: early diagnostic pointers and prognostic factors. J Laryngol Otol; 2003; 117:861-865.

18. Palejwala SK, Zangeneh TT, Goldstein SA, Lemole GM. (2016) An aggressive multidisciplinary approach reduces mortality in rhinocerebral mucormycosis. Surg Neurol Int; 7:61-63.

19. Rao VM, El-Noveam KI. (1998) Sinonasal imaging: anatomy and pathology. Radiologic Clinics of North America; 36: 921-939.

20. Jung SH, Kim SW, Park CS, Song CE, Cho JH, et al. (2009) Rhinocerebral mucormycosis: consideration of prognostic factors and treatment modality. Int J Oral HNS; 36:274-279.

21. Kumar Nilesh, V Aaditee. (2018) Mucormycosis of maxilla following tooth extraction in immunocompetent patients: Reports and review. J Clin Exp Dent; 10: 300-305. 